\begin{tabular}{|c|c|c|}
\hline \multirow{3}{*}{$\begin{array}{l}\text { ITC 4/48 } \\
\text { Information Technology } \\
\text { and Control } \\
\text { Vol. 48/ No.4 / } 2019 \\
\text { pp. 637-647 } \\
\text { DOI 10.5755/j01.itc.48.4.23031 }\end{array}$} & \multicolumn{2}{|c|}{$\begin{array}{c}\text { Research on Intention Recognition Method Based on } \\
\text { Radial Basis Function Neural Network }\end{array}$} \\
\hline & Received 2019/03/27 & Accepted after revision 2019/10/16 \\
\hline & \multicolumn{2}{|c|}{ Crossef http://dx.doi.org/10.5755/j01.itc.48.4.23031 } \\
\hline
\end{tabular}

\title{
Research on Intention Recognition Method Based on Radial Basis Function Neural Network
}

\section{Han Yan, Han Ming, Ruoxi Yang}

College of Mechanical Engineering, Hebei University of Technology, Tianjin 300132, China; e-mails:201611201013@stu.hebut.edu.cn,201711201004@stu.hebut.edu.cn,201811201015@stu.hebut.edu.cn

\section{Tiejun Li}

College of Mechanical Engineering, Hebei University of Technology, Tianjin 300132, China; e-mail: Li_tiejun@hebut.edu.cn

Corresponding author: Li_tiejun@hebut.edu.cn

A robot should be endowed with certain collaboration experience to recognize human's behavioral intention. This paper provides a method based on machine learning to recognize the collaborator's intention. A radial basis function neural network model was built for offline practice of a robot to recognize intention. Some collaboration skills can be obtained by the robot by building a map between the collaborator's intention and the system state, deducing human's intention based on the dynamic characteristics of collaborator and robot and taking the collaborator's intention as the feedforward information for controlling the robot so as to estimate the human's intention online based on collaborator's force and robot's motion characteristics during collaboration. The proposed method can overcome the difficulties in building the human-robot collaboration model by traditional method, especially the complicated human motion model, and difficulties in estimation of impedance parameters of human body. An experiment was conducted on a motion platform with single degree of freedom. The results prove that the collaborator's force is reduced while synchronization of human-robot collaboration is improved, so that the compliance of collaborated motion is also improved.

KEYWORDS: Machine Learning, Radial Basis Function Neural Network, Human-Robot Collaboration, Intention Recognition. 


\section{Introduction}

The collaborative robot enjoys a history of more than 20 years. At present, it has a very wide space for application in military, aerospace, rehabilitation, nursing, industry, living, etc. The robot should be capable of sensing the force, judging the intention and making the decision of motion during the collaboration with human for handling, assembling, limb rehabilitation exercising, robot assisted walking and other work to ensure its safety, compliance and high efficiency.

For a long time, the human-robot collaboration system had an obvious defect, i.e. a robot calculates the position information based on the acquired information, then transmits the position information to its controller, and finally realizes the follow motion, resulting in the time delay [6]. In order to solve this problem, Maeda et al. [12], on the basis of human-human collaboration, put forward that if a robot is endowed with the prejudging ability like human, can recognize the collaborator's intention in real time and build the relationship between its expected trajectory and the collaborator's intention based on certain rule, many problems can be solved, i.e. the robot and the collaborator are not synchronous, and the collaborator's force is too high. Finally, the robot can make more natural and more flexible motion. Therefore, the expected trajectory of a robot should be designed as a random variable related to the collaborator's intention. The recognition of collaborator's intention becomes an important task. At present, the researches on methods of collaborator's intention recognition mainly focus on the methods based on model and machine learning. Human intentions come from the brain. The research should start from the root of generating the intention. If the human's intention signal can be abstracted from brain, the human's intention can be clear and can be transmitted to the effector by external signal. For example, a neurologist, prof. Gregoire Courtine [4] from Swiss Federal Institute of Technology took an experiment on a monkey whose nervus centralis of leg is injured. The brain signal was abstracted from monkey's brain and transmitted to the effector (monkey's leg) by external transmission mode. The paralyzed monkey regained the ability to walk. At present, the brain signal is mainly abstracted by wearing the wearable devices. However, the head wearable device cannot abstract the brain signal well and the interference may not be eliminated. In addition, the correspondence relationship between brain phonetics and action is fuzzy. Thus, the research has not made significant progress.

Many researchers simplify the control model of human's upper limbs as the impedance control model, even directly simplify as the mass-spring-damper system and represent the human's intention as a virtual variable $[14,20,5]$ of expectation. The method has an obvious defect, i.e. the method is based on a hypothesis that the mass, damper and rigidity of a collaborator are not change during the human-robot collaboration. However, collaborator's intention always changes with the change of current scenes, goals and tasks, i.e. the human's intention changes at any time. Therefore, the method of recognition of collaborator's intention based on model is not applicable to human-robot collaboration system with strong randomness. Thus, the prediction model built by machine learning method without consideration of human body model can make the robot recognize the collaborator's intention more visually and effectively [3].

Among the current research results, Lee et al. [10] practiced the Hidden Markov Model (HMM) by Baum-Welch algorithm and predicted the collaborator's action and gesture by learning the meaning of collaborator's gesture. Recognition of collaborator's intention by visual information is an effective method, including face recognition, strength or gradient recognition, contour recognition, etc. $[9,21]$ The collaborator's intention can be recognized by posture language of human body [19]. For example, Lv et al. [11] recognized the human body's motion behavior on plane. Mikolajczyk et al. [13] recognized the collaborator's behavior based on some appearance features. Human's behavior mode may be corresponding to the human's intention. The human's intention can be regarded as the hidden state in HMM. The collaborator's intention can be indirectly recognized by observation of collaborator's behavior [16]. At present, this opinion becomes an important research idea in the intention recognition field. Tahb [18] and Weede et al. [17] adopted Dynamic Bayesian Network and Hybrid Dynamic Bayesian Network method to recognize the collaborator's intention, respectively. Jeon et al. [7, 22] adopted Ontology, Graph and Utility method 
to recognize the collaborator's intention. Kelley et al. [8] adopted a novel HMM to recognize the collaborator's intention, while this method was analyzed by M Awais et al. [2] by Finite State Machines method based on probability.

Among above methods, most of them are based on gestures or actions and deduce the collaborator's intention on the basis of human's action without consideration of necessity of using mutual information and state information of the collaboration system in human-robot collaboration. Thus, these methods cannot meet the demand of human-robot collaboration. Therefore, this paper provides a method based on machine learning to recognize the collaborator's intention on the basis of the human-robot interaction information. A radial basis function neural network (RBFNN) model was built for offline practice of a robot to recognize intention. Some collaboration skills can be obtained by the robot by building a map between the collaborator's intention and the system state, deducing human's intention based on the dynamic characteristics of collaborator and robot and taking the collaborator's intention as the feedforward information for controlling the robot so as to estimate the human's intention on line based on collaborator's force and robot's motion characteristics during collaboration.

\section{The Principle of Intention Recognition Based on Radial Basis Function Neural Network}

The human's intention is embodied by dynamic performance of system and can be estimated on the ground of external performance of the system. Based on this idea, the intention recognition method based on radial basis function neural network is put forward. Its principle is shown in Fig. 1. The intention identification process includes offline learning and online real-time estimation. The human's intention is simplified as the speed of interaction point between the collaborator and the robot $\left\{\dot{x}_{\text {hd }}\right\}$. Ge et al. [6] proved the rationality of this hypothesis. In order to prevent "resilience" of the robot, the expected rigidity of robot is set as 0, i.e. $K_{\mathrm{d}}=0$. If there is no requirements for expected inertia of robot, only the expected speed of ro-

\section{Figure 1}

A schematic diagram of intention recognition based on radial basis function neural network

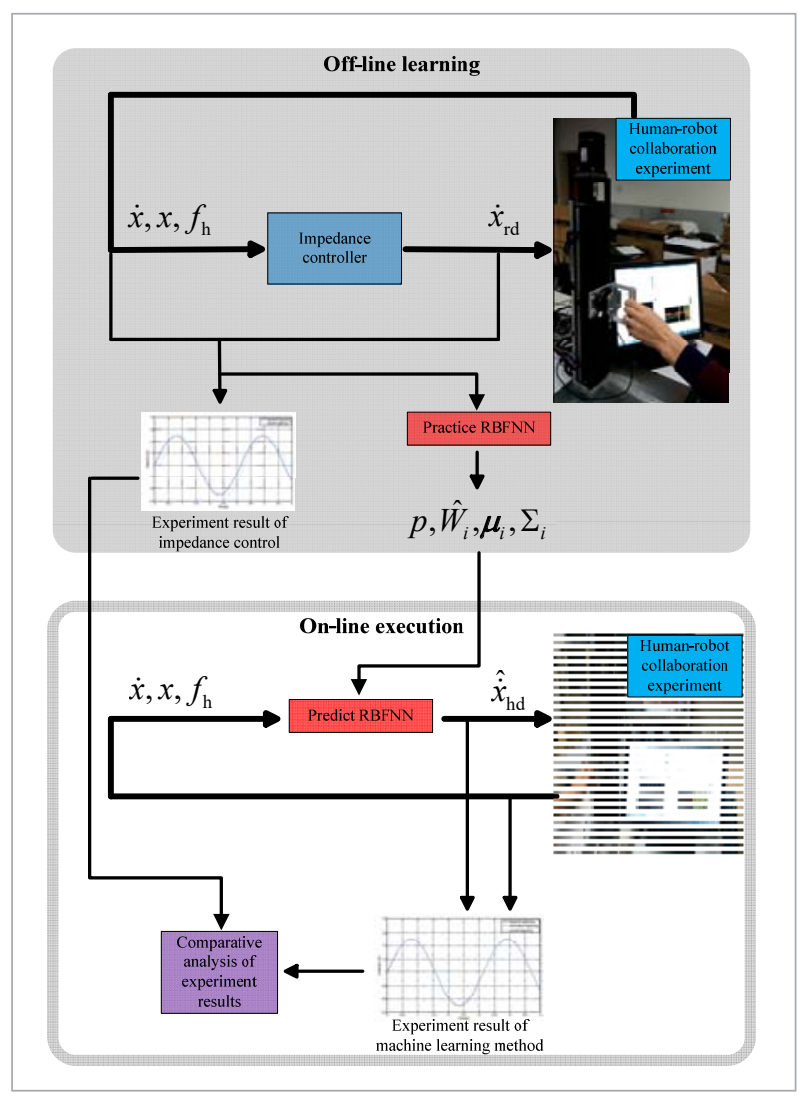

bot $\left\{\dot{x}_{\mathrm{rd}}\right\}$ affects the interaction in impedance control. Therefore, the human's intention is defined as the speed of collaborator's palm, and the expected speed of robot $\left\{\dot{x}_{\text {rd }}\right\}$ verges on the human's intention speed $\left\{\dot{x}_{\mathrm{hd}}\right\}$ in an optimal mode to realize the synchronous motion of human-robot collaboration and play the role of reducing the interaction force.

In this paper, robot perceives human's intention by the force from human, and follows human's intention to move. In this process, RBFNN is used to predict human behavior. RBFNN has strong input and output mapping ability and it can avoid falling into local minimum when training. Poggio et al. [15] have proven that the RBFNN is superior in approximating continuous functions. The essence of RBFNN used here is to build human intent model based on human historical behavior data including the speed, position and the corresponding interaction force of the robot. 
The Gaussian function has good local approximation capability and is adopted as the radial basis transmission function to predict the collaborator's intention $\left\{\hat{\dot{x}}_{\mathrm{hd}}\right\}$. Therefore, the output layer of radial basis function neural network is one layer and is expressed as:

$$
y=\sum_{i=1}^{p} w_{i} \exp \left(-\frac{1}{2 \sigma^{2}}\left\|x-c_{i}\right\|^{2}\right), i=1,2, \cdots p,
$$

where, $y$ is the output of neural network, $p$ is the size of hidden layer, $W_{i}$ is the weight from hidden layer to output layer, $\sigma$ is the extension constant of radial basis function, $x$ is the input of neural network and $c_{i}$ is the center of neural network basis function.

In order to ensure the effective and real-time intention recognition, only the end-speed, position and interaction force are selected as the intention estimation information. The network parameters are defined by combining the variable of human-robot collaboration system. Name and description of parameters are as follows:

$$
D=\left\{\left(\dot{x}_{\mathrm{hd}}^{(i)}, \dot{x}^{(i)}, x^{(i)}, f^{(i)}\right)\right\}, i=1 \cdots n,
$$

where, $\dot{x}_{\mathrm{hd}}^{(i)}$ is the vector of human's intention in the $i$ th group of observation data; $\dot{x}^{(i)}$ is the vector of robot's end-speed in the $i$ th group of observation data; $x^{(i)}$ is the vector of robot's end-position in the $i$ th group of observation data; $f^{(i)}$ is the vector of interaction force between the robot and the collaborator in the $i$ th group of observation data and the vector of force in motion direction which triggers the sensor.

\section{Basis Function Center Design Method Based on K-means}

The prerequisite of estimation of collaborator's intention based on radial basis function neural network is building a proper neural network model. The key points of design lie in the method of selecting hidden layer node based on data and the design method of weight from hidden layer to output layer. Due to the number of hidden nodes in radial basis function neural network, i.e. $p$ in formula 1 , greatly influences the generalization ability of the whole network, it is very important to find a rational method to confirm the number of hidden nodes. There are many methods to confirm the hidden nodes, such as clustering method, gradient training method and resource allocation network. However, in practice, the dynamic clustering algorithm is often adopted for data center selection by self-organization. During learning, the position of data center should be dynamically adjusted.

$\mathrm{K}$-means clustering method, as a common and effective clustering method, can confirm the extension constant of hidden nodes on the basis of the distance among various clustering centers. The main idea of this method is that the $\mathrm{K}$ value and $\mathrm{K}$ initial class cluster centers should be set firstly, then each center point should be allocated in the class cluster where there is the class cluster center which is closest to the center. After this, the mean of class clusters should be recalculated according to the data in each class cluster. It is a process of allocating center points and updating the class cluster centers by iteration method. The algorithm will not stop until the change of class cluster center is very small (convergence) or the specified iteration number are achieved. Rational determination of $K$ value and $K$ initial class cluster centers greatly influences of the clustering effect. Therefore, $K$ value and $K$ initial class cluster centers shall be selected through calculation.

In practical application, $K$ value may not be large and can be determined by enumeration. It is known from literature [1] that if the set class cluster number equals to or is more than the proper class cluster number, the radius or diameter of class cluster will change slowly. If the set class cluster number is smaller than the proper class cluster number, the radius or diameter of class cluster will change rapidly.

Choosing the value $K$ by enumeration can get the optimal result. At the same time, it can get the Weighted mean value, $W$ of mean centroid distance (radius) of class cluster based on the determined indexes. The index is expressed as follows:

$$
W=\sum_{i=1}^{k} \operatorname{mean}\left(r_{i}\right) \times \frac{n_{i}}{N},
$$

where, $k$ is the number of class cluster, mean $\left(r_{i}\right)$ is the mean radius of the $i$ th class clusters, $n_{i}$ is the number of data points in the the $i$ th class clusters, $N$ is the total number of data to be classified. 


\section{Network Weight Design Based on Gradient Descent Method}

The gradient descent algorithm, also called steepest descent algorithm, as an effective optimization algorithm, plays an important role in iteration process of back-propagation algorithm. Therefore, the gradient descent method can be combined with the backpropagation idea to solve the weight from hidden layer to output layer of neural network. Suppose that there are $m$ groups of fixed sample sets $\left\{\left(\mathrm{x}^{(1)}, \mathrm{y}^{(1)}\right),\left(\mathrm{x}^{(2)}, \mathrm{y}^{(2)}\right), \cdots,\left(\mathrm{x}^{(i)}, \mathrm{y}^{(i)}\right), \cdots,\left(\mathrm{x}^{(m)}, \mathrm{y}^{(m)}\right)\right\}_{i=1 \cdots m}$. The entire cost function can be defined as:

$$
J(W)=\frac{1}{2} \sum_{i=1}^{m}\left(h_{W}\left(x^{(i)}\right)-y^{(i)}\right)^{2},
$$

where, $h_{W}(\cdot)$ is the function model related to weight, the purpose is to obtain $W=\left(w_{1} w_{2} \cdots w_{i} \cdots w_{m}\right)_{i=1 \cdots m}$ by backpropagation algorithm to minimize $J(W)$. The convergence threshold $\varepsilon$ of gradient descent algorithm should be set. To solve above formula to minimize the objective function $J(W)$, finding the direction with fastest gradient descent, the partial derivative of Equation (4) can be obtained as:

$$
\frac{\partial}{\partial W} J(W)=\frac{\partial}{\partial W} \frac{1}{2} \sum_{i=1}^{m}\left(h_{W}\left(x^{(i)}\right)-y^{(i)}\right)^{2}=\left(h_{W}(x)-y\right) x^{(i)} .
$$

In addition, the step length $\alpha$ in gradient descent direction, i.e. variation along the gradient descent direction each time, should be set. The descent process is expressed in Equation (6):

$$
W_{i}=W_{i}-\alpha \frac{\partial}{\partial W} J(W)=W_{i}-\alpha\left(h_{W}(x)-y\right) x^{(i)} .
$$

The above calculation can determine the weight from hidden layer to output layer.

\section{Design of Impedance Controller}

Resilience force should not be set in human-robot collaboration. Therefore, the robot impedance control rigidity is set as 0, i.e. $K_{d}=0$. In cartesian space, the rotor impedance control can be expressed as:
$M_{\mathrm{d}} \ddot{x}+B_{\mathrm{d}}\left(\dot{x}_{\mathrm{rd}}-\dot{x}\right)=-f+f_{\mathrm{d}}$,

where, $M_{\mathrm{d}}$ and $B_{\mathrm{d}}$ are expected mass matrix and damping matrix, respectively, $\ddot{x} \dot{x}$ and $\dot{x}_{\text {rd }}$ are end-acceleration, speed and expected end-speed of robot, $f$ is the actual force and $f_{\mathrm{d}}$ is the expected interaction force. In order to realize synchronization, the expected endspeed of the robot should be synchronous with the human's intention $\left(\dot{x}_{\mathrm{rd}}=\dot{x}_{\mathrm{hd}}\right)$, and the end-speed of robot should follow the expected speed $\dot{x}=\dot{x}_{\text {rd }}$, i.e. we wish the follow deviation of robot is 0 .

In order to improve the follow effect of end-trajectory of the robot, the designed impedance controller is shown in Figure 2. The speed compensation should be continuously adjusted on the basis of difference between the human-robot interaction contact force and the expected force to make the end-speed follow the expected end-speed well. The impedance controller:

$M_{\mathrm{d}} \ddot{x}+B_{\mathrm{d}}\left(\dot{x}_{\mathrm{rd}}-\dot{x}+\Omega\right)=-f+f_{\mathrm{d}}$,

where, $\Omega$ is the speed compensation, $f_{\mathrm{d}}$ is same with above, i.e. $f_{\mathrm{d}}=[-t, t], t$ is the constant which is approximate to 0 in positive direction. Other parameters are the same as indicated above. The speed compensation is set as:

$\Omega(t)=\Omega(t-\lambda)+\eta \frac{\left(f_{\mathrm{d}}(t-\lambda)\right)-(f(t-\lambda))}{b}$,

where, $\lambda$ is the sampling cycle of controller, $\eta$ is update rate and $b$ is a constant.

Figure 2

A schematic diagram of impedance control

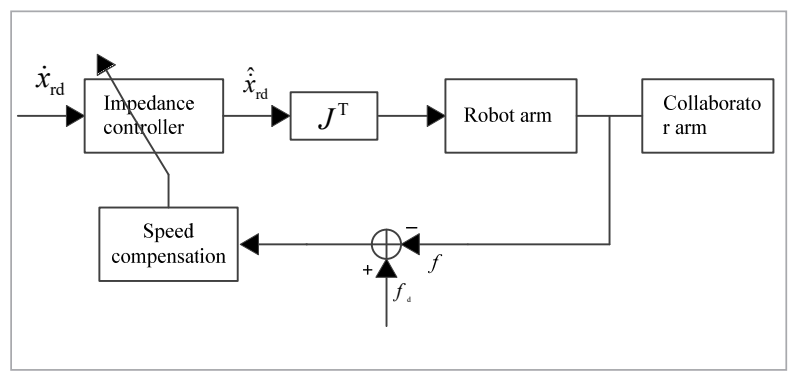




\section{Experiment}

\subsection{Experiment Devices}

In order to verify the effectiveness of the proposed method, a translational single-degree-of-freedom robot system was selected for collaboration with human to avoid the influence of complicated experiment device and control coupling of motion with multi-degree of freedom. The schematic diagram and picture of interaction system are shown in Fig. 3. The parameters of the system are shown in Table 1 . The interaction force is acquired by the $6 \mathrm{D}$ force sensor installed at the end of the robot. Here, only the force information in the sliding table moving direction is extracted. The operation frequency of the whole system is $1 \mathrm{kHz}$.

\section{Figure 3}

A schematic diagram and picture of human-robot collaboration system

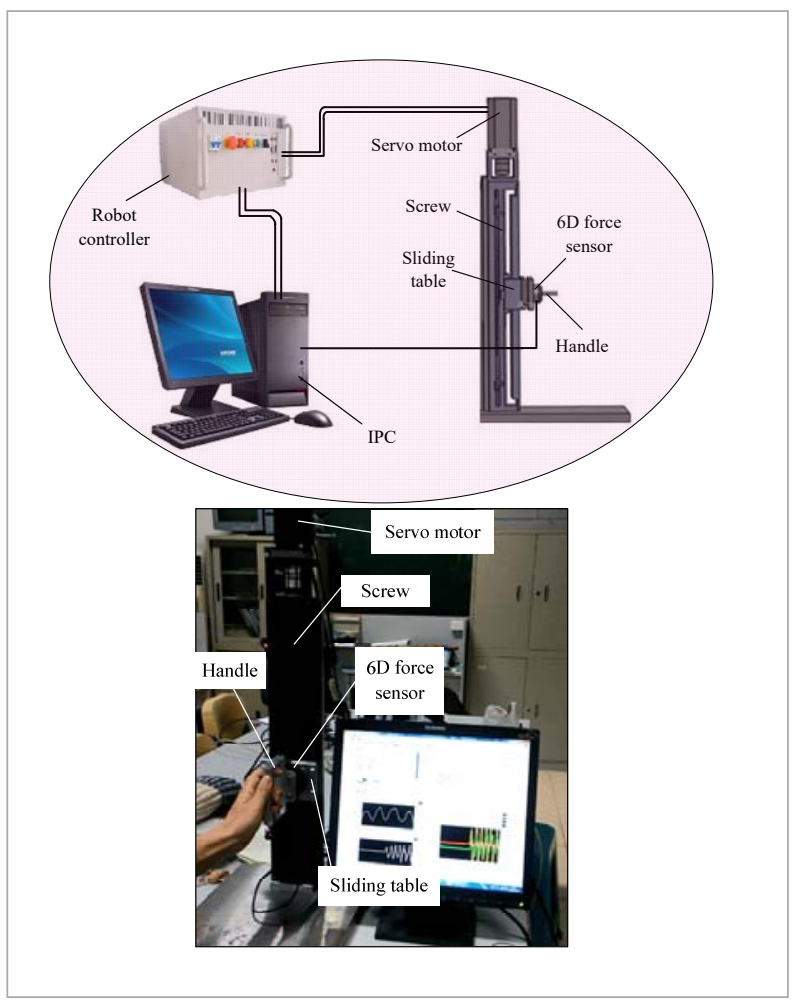

The task performance is that the end (sliding table) of robot is synchronous with the hand which is installed with the force sensor and the collaborator's hand moves at random.
Table 1

Important parameters of human-robot collaboration system

\begin{tabular}{c|c}
\hline Name & Parameter \\
\hline IPC & Advantech IPC-610 \\
\hline Robot controller & ADLINK PCI-8258 \\
\hline 6D force sensor & ATI-SI125 \\
\hline $\begin{array}{c}\text { Servo motor and } \\
\text { driver }\end{array}$ & $\begin{array}{c}\text { Yaskawa AC servo driver 400W, } \\
\text { rated torque of motor } 1.27 \mathrm{~N} . \mathrm{m}\end{array}$ \\
\hline
\end{tabular}

The acquired data is used for practicing the radial basis function neural network. The data acquisition flow chart (Figure 4) was established for the experiment device of human-robot collaboration system.

Figure 4

Data acquisition flow chart



\subsection{Sample Data Acquisition Based on Impedance Control}

Firstly, the force sensor and the robot were initialized. In order to unify the end position information acquired each time, the robot should move to the position Home. In the data acquisition, the robot in the human-robot collaboration system was controlled by the impedance control mode. It is worth noting that in order to maximize the coverage of motion space of human-robot collaboration by the acquired data and to make the practiced sample more representative, the collaborator should move at different speeds within the motion space of the robot during the data acquisition. For example, collaborator can move the handle 
under the sinusoidal speed change rule. During the whole data record process, the operation frequency is $1 \mathrm{kHz}$ and the sampling frequency is $50 \mathrm{~Hz}$. The whole sampling process lasts for 10s. Sampling should be repeated three times.

It can be seen from above analysis that the interaction force of the human-robot collaboration system with single degree of freedom is the 1D information $\left(f \in \mathrm{R}^{1}\right)$ and the sensor is accompanied with $10 \%$ noise. To acquire the end-position and end-speed of the robot, the value fed back by servo motor encoder should be adopted. Then, the end-position and endspeed can be obtained on the basis of conversion relationship between joint space and Cartesian space. The end-position and end-speed of the robot are both the 1 D data $\left(x \in \mathrm{R}^{1}\right.$ and $\left.\dot{x} \in \mathrm{R}^{1}\right)$, respectively. The collaborator's intention data $\dot{x}_{\text {hd }} \in \mathrm{R}^{1}$ can be acquired by Equation (8). Because only the intention recognition of human-robot collaboration system with single degree of freedom is verified, the human's intention information is also the $1 \mathrm{D}$ vector data in the experiment. In order to achieve the purpose of synchronization of human-robot collaboration, set $\dot{x}_{\text {rd }}=\dot{x}_{\text {hd }}$. In order to obtain the expected speed $\dot{x}_{\text {rd }}$ of robot in Equation (8), set the mass matrix and damping matrix of the robot as $M_{\mathrm{d}}=0.1$ and $f_{\mathrm{d}}=0.5 \mathrm{~N}$, respectively. Based on the above description of sampling cycle and repeated times, the practice data sample during $3 \times 50 \times 10=1500$ interaction process can be obtained.

\subsection{Estimation of $\mathrm{K}$ Value and Determination of Network Basis Function Center}

The data is clustered by $\mathrm{K}$-means algorithm. Because $\mathrm{K}$-means algorithm is very sensitive to class cluster number and initial class cluster center, the class cluster number and the initial class cluster center should be determined firstly. By observing the sample data acquired, four kinds of data in the sample (expected speed $\dot{x}_{\text {rd }}$ of robot, end-speed and position $\dot{x}, x$ of robot and interaction force $f$ ) are of the same order of magnitude. Therefore, there is no need to standardize the data if K-means algorithm is adopted. The sample data is classified into class 1-10 by enumeration. Some clustering results are shown in Figure 5.

In order to determine $K$ value, the weighted means of mean centroid distance (radius) of all class clusters for different class cluster quantities are calculated respectively based on the Equation (6). The correspon-
Figure 5

Some clustering effects if $k$ is different

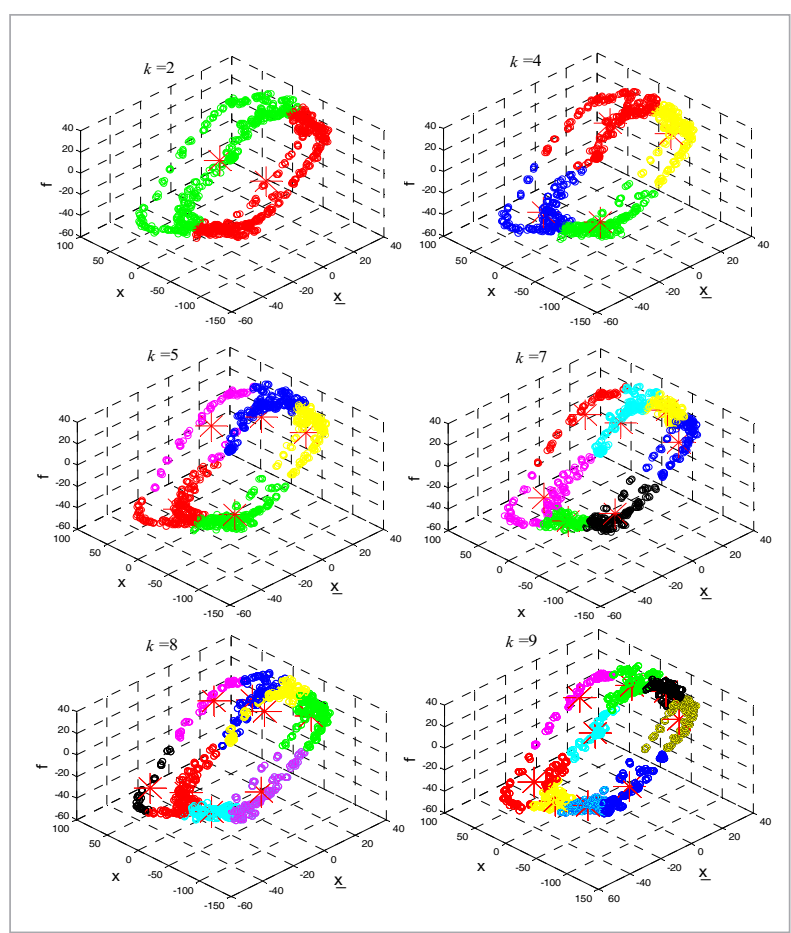

dence relationship between the class cluster number $K$ and the calculated weighted means is built, as shown in Figure 6.

\section{Figure 6}

Class cluster number $k$ and weighted means of mean centroid distance of class clusters

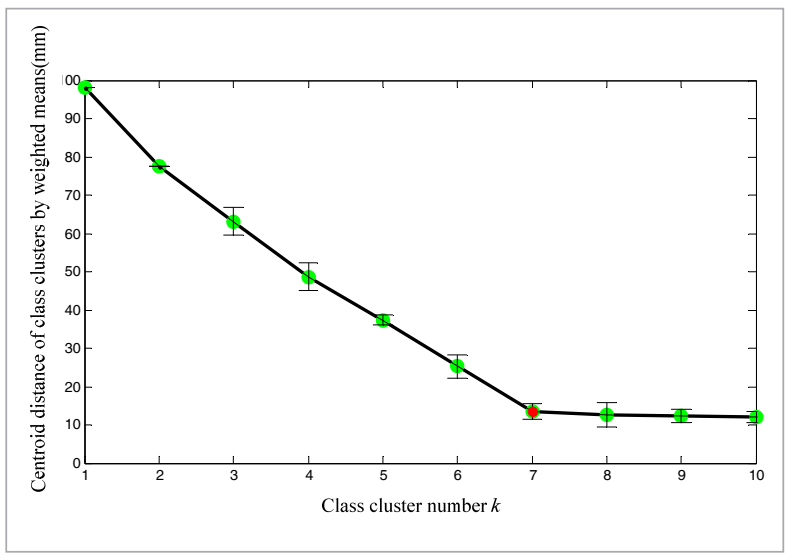

It can be observed clearly from Figure 6 that in front of class 7 , the change is obvious, the weighted mean descends rapidly; behind class 7 , weighted mean is 
stable and the change is slow. If $k$ is $\%$, there is the turning point of weighted means of mean centroid distance of all class clusters. According to [1], the acquired sample data should be classified into 7 classes, i.e. $k=7$. Red mark $※$ in Figure 5 represents the class cluster center. In case of $k=7$, the marked position is regarded as the center of basis of radial basis function neural network. The obtained center positions and Gaussian function variances are shown in Table 2.

\section{Table 2}

Mean center coordinates of class clusters and Gaussian function variances

\begin{tabular}{|c|c|c|c|c|}
\hline $\begin{array}{l}\text { Class } \\
\text { cluster }\end{array}$ & \multicolumn{3}{|c|}{$\begin{array}{l}\text { Mean center coordinates of class } \\
\text { clusters }\end{array}$} & $\begin{array}{l}\text { Gaussian } \\
\text { function } \\
\text { variances }\end{array}$ \\
\hline 1 & -6.7376 & 8.4750 & -9.8347 & 10.7798 \\
\hline 2 & 14.9258 & -84.8704 & 19.1362 & 7.5946 \\
\hline 3 & 21.8041 & 26.2799 & 19.8990 & 11.2995 \\
\hline 4 & -21.1810 & 55.1060 & -24.5560 & 7.9990 \\
\hline 5 & 28.7029 & -31.0898 & 28.2646 & 9.0735 \\
\hline 6 & -24.7955 & -75.1356 & -22.5454 & 9.4053 \\
\hline 7 & -34.2421 & $-15.55^{r} 74$ & -34.4726 & 7.4068 \\
\hline
\end{tabular}

The weight is determined by the gradient descent algorithm. The learning speed $\alpha$ of gradient descent algorithm is set to 0.1. The change of weight is shown in Figure 7 . With the increase of iteration number, the weight from hidden layer to output layer gradually converges. The prediction precision $\varepsilon$ is set to $10^{-6}$. The radial basis function neural network weight is shown in Table 3.

\section{Table 3}

Weight from hidden layer to output layer

\begin{tabular}{c|c|c|c}
\hline $\begin{array}{c}\text { Hidden layer } \\
\text { sequence } \boldsymbol{i}\end{array}$ & Weight $\omega_{i}$ & $\begin{array}{c}\text { Hidden layer } \\
\text { sequence } \boldsymbol{i}\end{array}$ & Weight $\omega_{\boldsymbol{i}}$ \\
\hline 1 & 14.7179 & 5 & 38.1925 \\
\hline 2 & 11.0514 & 6 & -21.5968 \\
\hline 3 & 27.6283 & 7 & -50.2440 \\
\hline 4 & -9.2611 & & \\
\hline
\end{tabular}

\subsection{An Analysis of Human-Robot Motion Synchronization and Collaborator's Interaction Force}

In order to verify that the intention recognition method based on radial basis function neural network proposed in this paper is more excellent in synchronization of man-robot motion, the experiment was conducted by impedance control method and the proposed method. The speed tracking curves of these two methods were drawn respectively, as shown in Figures 7-8.

\section{Figure 7}

Speed tracking curve of method based on impedance control

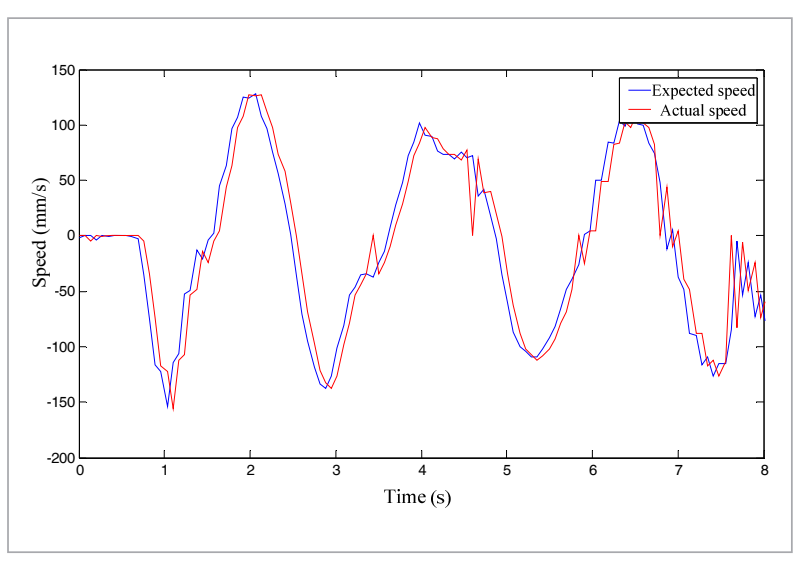

\section{Figure 8}

Speed tracking curve of method based on radial basis function neural network

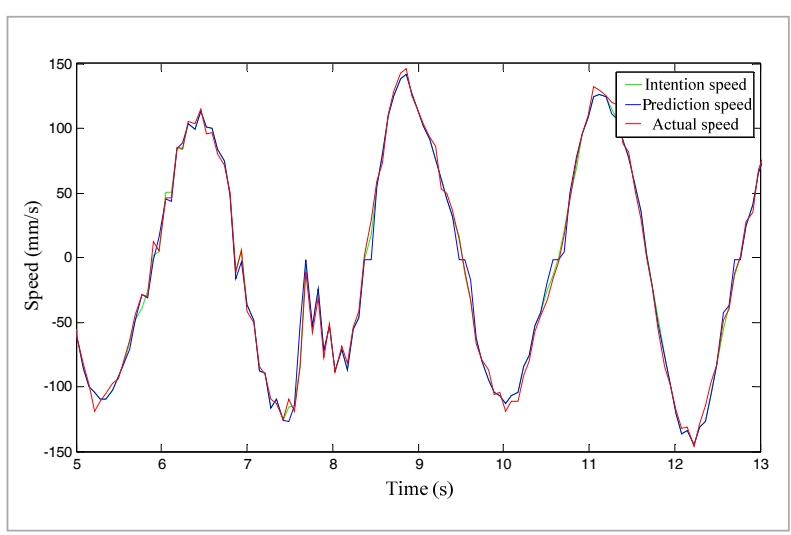

It can be seen from Figure 7 that the actual speed of robot is obviously slower than the expected speed during human-rotor collaboration if the impedance control method is adopted. The inherent delay characteristics of impedance control restricts the syn- 
chronization of human and robot. The robot is always in the passive follow state.

It can be seen from Figure 8 that compared with impedance control method, the intention recognition method based on radial basis function neural network can eliminate the delay caused by impedance control method by predicting the collaborator's intention on the basis of current human-rotor collaboration state (intention speed in Figure 8). The proposed method realizes the synchronization of human-robot motion.

In order to further explain the role of the proposed intention recognition method in reducing the collaborator's interaction force and make the robot finish the task more easily, the experiment was conducted by impedance control method and the proposed method. During interaction, the collaborator tried to keep the consistence of end-speed and motion trajectory. The interaction force information of the collaborator was abstracted, as shown in Figure 9.

It can be seen from Figure 9 that compared with the impedance control method, the intention recognition

\section{Figure 9}

Curve of change of interaction force of method based on impedance control and radial basis function neural network

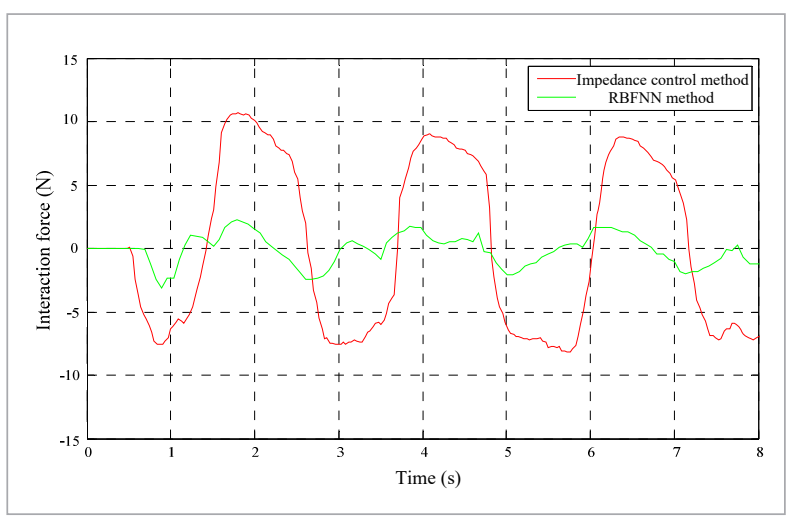

method based on radial basis function neural network can greatly reduce the collaborator's force applied on the system and has obvious effect. In order to further explain that the proposed method can reduce the interaction force during the human-robot collaboration and improve the compliance of the robot, the fragile object (egg roll) was used to push the handle of experiment device for verification. The experimental scene is shown in Figure 10. The experiment result shows that the proposed method can ensure the successful
Figure 10

Effectiveness verification experiment with fragile object

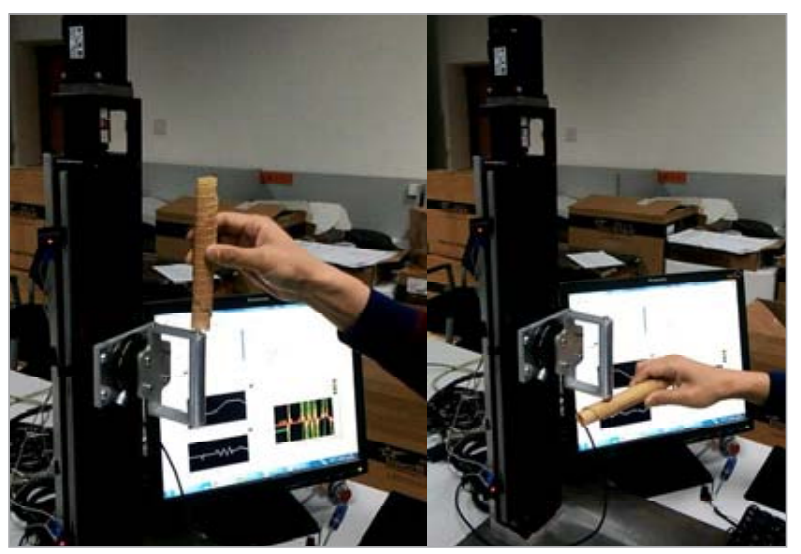

human-robot collaboration without damage of the fragile object.

\section{Conclusion}

A machine learning based method (radial basis function neural network model) was adopted in this paper to recognize the collaborator's intention during human-robot collaboration. Firstly, in order to acquire the practice data of neural network, the human-robot collaboration system with single degree of freedom was adopted. The sample data was acquired by impedance control method. Secondly, the neural network parameters were identified by offline mode and the radial basis function neural network model was built. Finally, the collaborator's intention can be recognized by the online prediction mode. In order to explain the effectiveness of the machine learning method, the endspeed of robot, interaction force and other data during the human-robot collaboration system were analyzed and the proposed method and the impedance control method were compared and analyzed. The experiment result shows that the proposed method can accurately recognize the collaborator's intention, improve the synchronization of human-robot collaboration motion, effectively reduce the collaborator's force during interaction process, reduce the collaborator's effort, make the human-robot collaboration more easy, improve the compliance of the robot, endow the certain human-robot collaboration ability to the robot, and finally improve the intelligence of the robot. 


\section{References}

1. Anand, R., Jeffrey, D. U. Mining of Massive Datasets. In Wang, B. (Eds), Mining and Distributed Processing of Large Scale Data in Internet, Posts and Telecom Press: Beijing, 2012.

2. Awais, M., Henrich, D. Human-Robot Collaboration by Intention Recognition Using Probabilistic State Machines. Proceedings of the 19th International Workshop on Robotics in Alpe-Adria-Danube Region, (RAAD 2010), Balatonfured, Hungary, June 26-27, 2010, 75-80. https://doi.org/10.1109/RAAD.2010.5524605

3. Awais, M., Henrich, D. Online Intention Learning for Human-Robot Interaction by Scene Observation. Proceedings of the 2012 IEEE Workshop on Advanced Robotics and its Social Impacts, (ARSO 2012), Munich, Germany, May 21-23, 2012, 13-18. https://doi. org/10.1109/ARSO.2012.6213391

4. Capogrosso, M., Milekovic, T., Borton, D., Wagner, F., Moraud, E. M. A Brain-Spine Interface Alleviating Gait Deficits after Spinal Cord Injury in Primates. Nature, 2016, 539(7628), 284-288. https://doi.org/10.1038/nature20118

5. Erickson, D., Weber, M., Sharf, I. Contact Stiffness and Damping Estimation for Robotic Systems. International Journal of Robotics Research, 2003, 22(1), 41-57. https://doi.org/10.1177/0278364903022001004

6. Ge, S. S., Li, Y., He, H. Neural-Network-Based Human Intention Estimation for Physical Human-Robot Interaction. Proceedings of 8th International Conference on Ubiquitous Robots and Ambient Intelligence, (URAI 2011), Incheon, Korea, November 23-26, 2011, 390-385. https://doi.org/10.1109/URAI.2011.6145849

7. Jeon, H., Kim, T., Choi, J. Ontology-Based User Intention Recognition for Proactive Planning of Intelligent Robot Behavior. Proceedings of the 2008 International Conference on Multimedia and Ubiquitous Engineering, (MUE 2008), Busan, Korea, April 24-26, 2008, 244-248. https://doi.org/10.1109/MUE.2008.97

8. Kelley, R., Tavakkoli, A., King, C., Nicolescu, M., Nicolescu, M., Bebis, G. Understanding Human Intentions via Hidden Markov Models in Autonomous Mobile Robots. Proceedings of the 3rd ACM/IEEE International Conference on Human-Robot Interaction, (HRI 2008), Amsterdam, Netherlands, March 12-15, 2008, 367-374. https://doi.org/10.1145/1349822.1349870

9. Ke, Y., Sukthankar, R., Hebert, M. Spatio-Temporal Shape and Flow Correlation for Action Recognition. Proceedings of the 2007 IEEE Computer Society Conference on Computer Vision and Pattern Recognition, (CVPR 2007), Minneapolis, United states, June 17-22, $200 \%$. https://doi.org/10.1109/CVPR.2007.383512

10. Lee, C., Xu, Y. Online, Interactive Learning of Gestures for Human/Robot Interfaces. Proceedings of the 1996 13th IEEE International Conference on Robotics and Automation, (ICRA 1996), Minneapolis, United states, April 22-28, 1996, 2982-2987. https://doi.org/10.1109/ ROBOT.1996.509165

11. Lv, F., Nevatia, R. Single View Human Action Recognition Using Key Pose Matching and Viterbi Path Searching. Proceedings of the 2007 IEEE Computer Society Conference on Computer Vision and Pattern Recognition, (CVPR 2007), Minneapolis, United states, June 1722, 2007. https://doi.org/10.1109/CVPR.2007.383131

12. Maeda, Y., Hara, T., Arai, T. Human-Robot Cooperative Manipulation with Motion Estimation. Proceedings of the 2001 IEEE International Conference on Intelligent Robots and Systems, (IROS 2001), Maui, HI, United states, October 29-November 3, 2001, 2240-2245. https://doi.org/10.1109/IROS.2001.976403

13. Mikolajczyk, K., Uemura, H. Action Recognition with Motion-Appearance Vocabulary Forest. Proceedings of the 26th IEEE Conference on Computer Vision and Pattern Recognition, (CVPR 2008), Anchorage, United states, June 23-28. https://doi.org/10.1109/ CVPR.2008.4587628

14. Modares, H., Ranatunga, I., Lewis, F. L., Popa, D. O. Optimized Assistive Human-Robot Interaction Using Reinforcement Learning. IEEE Transactions on Cybernetics, 2016, 46(3), 655-667. https://doi.org/10.1109/ TCYB.2015.2412554

15. Poggio, T., Girosi, F. Networks for Approximation and Learning. Proc IEEE, 1990, 78(9), 1481-1497. https:// doi.org/10.1109/5.58326

16. Poppe, R. A Survey on Vision-Based Human Action Recognition. Image and Vision Computing, 2010, 28(6), 976-990. https://doi.org/10.1016/j.imavis.2009.11.014

17. Schrempf, O. C., Hanebeck, U. D. A Generic Model for Estimating User-Intentions in Human-Robot Cooperation. Proceedings of the Second International Conference on Informatics in Control, (ICINCO 2005), Barcelona, Spain, September 14-17, 2005, 251-256. https:// doi.org/10.5220/0001166002510256

18. Tahboub, K. A. Intention Recognition of a Human Commanding a Mobile Robot. Proceedings of the 2004 IEEE Conference on Cybernetics and Intelligent Systems, 
(ICCIS 2004), Singapore, December 1-3, 2004, 895900. https://doi.org/10.1109/ICCIS.2004.1460707

19. Thurau, C., Hlavác`, V. Pose Primitive Based Human Action Recognition in Videos or Still Images. Proceedings of the 26th IEEE Conference on Computer Vision and Pattern Recognition, (CVPR 2008), Anchorage, United states, June 23-28. https://doi.org/10.1109/ CVPR.2008.4587721

20. Tsuji, T., Morasso, P. G., Goto, K., Ito, K. Human Hand Impedance Characteristics During Maintained Postu- re. Biological Cybernetics, 1995, 72(6), 475-485. https:// doi.org/10.1007/s004220050150

21. Wang, L., Suter, D. Learning and Matching of Dynamic Shape Manifolds for Human Action Recognition. IEEE Transactions on Image Processing, 2007, 16(6), 16461661. https://doi.org/10.1109/TIP.2007.896661

22. Youn, S. J., Oh, K. W. Intention Recognition Using a Graph Representation. World Academy of Science, Engineering and Technology, 2007, 25, 13-18. 